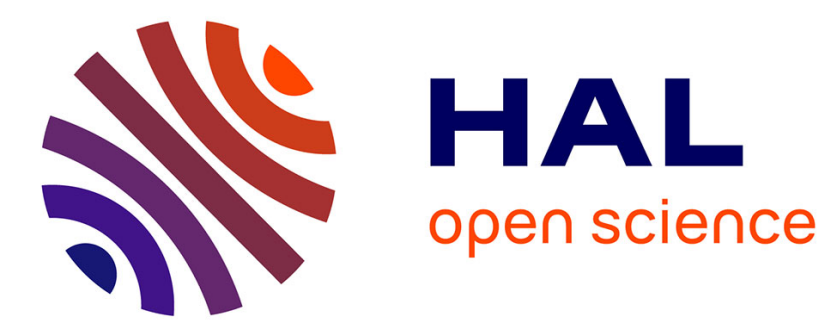

\title{
Défense et promotion des " droits des paysans" aux Nations unies : une appropriation oblique de l'advocacy par La Vía Campesina
}

Delphine Thivet

\section{- To cite this version:}

Delphine Thivet. Défense et promotion des "droits des paysans " aux Nations unies: une appropriation oblique de l'advocacy par La Vía Campesina. Critique Internationale, 2015, 67 (2), pp. 67-81. 10.3917/crii.067.0067 . hal-01161628

\section{HAL Id: hal-01161628 \\ https://hal.science/hal-01161628}

Submitted on 4 Apr 2018

HAL is a multi-disciplinary open access archive for the deposit and dissemination of scientific research documents, whether they are published or not. The documents may come from teaching and research institutions in France or abroad, or from public or private research centers.

$$
\text { Copyright }
$$

L'archive ouverte pluridisciplinaire HAL, est destinée au dépôt et à la diffusion de documents scientifiques de niveau recherche, publiés ou non, émanant des établissements d'enseignement et de recherche français ou étrangers, des laboratoires publics ou privés. 


\title{
Défense et promotion
} des "droits des paysans " aux Nations unies : une appropriation oblique de l'advocacy par La Vía Campesina

\author{
par Delphine Thivet
}

lors que le déclin de la paysannerie se poursuit dans les pays industrialisés et commence à apparaître dans les pays émergents, certains producteurs agricoles manifestent depuis une trentaine d'années, au Nord comme au Sud, la volonté de faire entendre une voix paysanne indépendante, capable de s'opposer au processus de libéralisation des échanges de produits agricoles dans le cadre des négociations commerciales menées au sein du GATT (General Agreement on Tariffs and Trade), puis de l'Organisation mondiale du commerce (OMC). La Vía Campesina (Voie paysanne) s'inscrit dans cette entreprise de « globalisation de la lutte $»^{1}$. Durant les quinze premières années de son existence, ce mouvement né en mai 1993 et qui revendique aujourd'hui plus de deux cents millions de membres, n'a eu de cesse d'élaborer et de coordonner des actions collectives transnationales contre la libéralisation des politiques agricoles ${ }^{2}$ tout en œuvrant à la consolidation

\footnotetext{
1. Le slogan de La Vía Campesina est «Globalisons la lutte ! Globalisons l'espoir ! ».

2. Notamment une marche anti-GATT à Genève (4-6 décembre 1993), la participation à des manifestations contre les politiques menées par le Fonds monétaire international (FMI) et la Banque mondiale à Madrid (2 octobre 1994), contre le GATT lors de la célébration de son cinquantenaire à Genève (mai 1998), contre l'OMC à Seattle (novembre 1999) ou à Hong-Kong (décembre 2005).
} 
de sa structure organisationnelle interne. Depuis le début des années 2000, La Vía Campesina est entrée dans une nouvelle phase de mobilisation, ses militant.e.s s'efforçant désormais d'investir les espaces onusiens et de transcrire la cause paysanne en des termes juridiques. Face à la violation massive des droits civils et politiques, mais aussi économiques et sociaux que subissent de nombreuses communautés rurales à travers le monde, le projet est né en effet de faire reconnaître l'existence de «droits des paysans » au niveau international. C'est ainsi que le 6 avril 2009, l'Indonésien Henry Saragih, alors coordinateur général de La Vía Campesina, s'est adressé à l'Assemblée générale des Nations unies à New York pour réclamer l'adoption par le Conseil des droits de l'homme d'une « Convention internationale sur les droits des paysannes et des paysans ».

Plusieurs chercheur.e.s ont étudié ce projet de Convention de La Vía Campesina. Parmi eux, les anthropologues Marc Edelman et Carwil James ont proposé en 2011 une analyse détaillée non seulement de son contenu mais aussi des obstacles juridiques et institutionnels ainsi que des facteurs macro-structurels susceptibles de faciliter ou au contraire d'entraver son adoption ${ }^{3}$. Dans un article de 2014, M. Edelman a retracé plus précisément les différentes étapes de l'élaboration de ce projet, depuis son origine indonésienne jusqu'au vote, en mars 2012, par le Comité consultatif du Conseil des droits de l'homme de la mise en place d'un groupe de travail intergouvernemental chargé de négocier un projet de « Déclaration des Nations unies sur les droits des paysans et autres personnes vivant dans les zones rurales $\gg^{4}$. Priscilla Claeys, quant à elle, s'est intéressée, à travers le cas de La Vía Campesina, à la manière dont les mouvements sociaux en général peuvent contribuer, sur un mode contre-hégémonique, à la création de « nouveaux droits humains $»^{5}$. Nous poursuivrons ici l'examen de la proposition de Déclaration/ Convention de La Vía Campesina en nous distanciant toutefois d'une approche centrée principalement sur des enjeux juridiques. Notre perspective sera plutôt celle d'une sociologie du militantisme transnational et, plus particulièrement, à travers l'étude de l'advocacy mis en œuvre par les membres de La Vía Campesina en faveur de ce nouvel instrument qu'est le droit international ${ }^{6}$, d'une sociologie des transformations des modes de contestation. Alors que les pratiques de

\footnotetext{
3. Marc Edelman, Carwil James, « Peasants' Rights and the UN System: Quixotic Struggle? Or Emancipatory Idea Whose Time Has Come? », Fournal of Peasant Studies, 38 (1), janvier 2011, p. 81-108.

4. M. Edelman, « Linking the Rights of Peasants to the Right to Food in the United Nations », Law, Culture and the Humanities, 10 (2), 2014, p. 196-211.

5. Priscilla Claeys, «The Creation of New Rights by the Food Sovereignty Movement: The Challenge of Institutionalizing Subversion », Sociology, 46 (2), 2012, p. 845.

6. La question des « droits paysans » n'est pas débattue seulement au Conseil des droits de l'homme. Le «droit à la terre et au territoire » et le « droit aux semences », par exemple, sont défendus par les membres de La Vía Campesina dans d'autres espaces institutionnels internationaux tels que la FAO : lors des négociations relatives au Traité international sur les ressources phytogénétiques pour l'alimentation et l'agriculture (TIRPAA, 2004); lors de la tenue de la Conférence internationale sur la réforme agraire et le développement rural (mars 2006 à Porto Alegre, Brésil) ; au sein du Comité de sécurité alimentaire lors des négociations relatives aux « Directives volontaires sur la gouvernance responsable des régimes fonciers » (mars 2012).
} 
plaidoyer tendent à devenir le nouveau format légitime et conforme de défense des causes des ONG depuis les années 19907, l'étude du cas de La Vía Campesina peut contribuer à enrichir la compréhension de la manière dont des formes de mobilisations plus discrètes et policées se diffusent de manière croissante et sont importées par des acteurs sociaux œuvrant à la défense de leurs intérêts au niveau transnational. Au-delà de la méfiance ressentie par certain.e.s de ses militant.e.s face au risque de dépossession induit par le recours à un répertoire d'action plus expert et institutionnel, le cas de La Vía Campesina permet de souligner les stratégies de mise à distance de certains acteurs sociaux qui s'approprient les techniques de l'advocacy d'une façon que l'on pourrait qualifier $\mathrm{d}^{\prime} \ll$ oblique $»^{8} \mathrm{car}$ sélective et désireuse de pérenniser le potentiel contestataire de la cause.

Nous soulignerons tout d'abord la spécificité de La Vía Campesina en rappelant le travail accompli par ses membres en vue de marquer leur différence, sur la scène internationale, dans le champ de la représentation de la cause paysanne. Nous retracerons ensuite la manière dont est apparu le projet de promouvoir et de défendre une « Convention internationale sur les droits des paysannes et des paysans » au niveau international et rendrons compte à la fois des potentialités de succès offertes par l'investissement des membres de La Vía Campesina dans cette stratégie d'action collective et des contraintes que celle-ci fait peser sur le mouvement. Enfin nous soulignerons quelques-unes des tensions qui en résultent quant à la possible déradicalisation de la cause ${ }^{9}$.

\section{Plaider en son nom propre, être expert de sa propre cause}

La Vía Campesina a été créée en avril 1992, au cours d'une réunion à Managua (Nicaragua), dans le cadre du Deuxième Congrès de l'UNAG (Unión Nacional de Agricultores y Ganaderos), de divers dirigeants d'organisations paysannes d'Amérique centrale, d'Amérique du Nord et d'Europe. Un an plus tard, en mai 1993, à Mons (Belgique), elle s'est elle-même définie officiellement comme un mouvement paysan international ayant pour but de permettre aux « petits et moyens paysans », sans-terres et autres agents marginalisés du monde rural de prendre en charge eux-mêmes la défense de leur cause. Depuis, le mouvement n'a cessé d'augmenter ses troupes : structuré en neuf secrétariats régionaux, un secrétariat international tournant, un Comité de coordination international et plusieurs groupes de travail thématiques, il est actuellement présent dans 73 pays et rassemble au total 164 organisations rurales, syndicats d'exploitants agricoles

7. Johanna Siméant, « Interpreting the Rise of International "Advocacy" », Humanity, 5 (3), 2014, p. 323-343.

8. Dans le sens utilisé par Richard Hoggart, La culture du pauvre. Étude sur le style de vie des classes populaires en Angleterre, Paris, Éditions de Minuit, 1970.

9. Cette étude se fonde sur le matériau recueilli depuis 2009 dans le cadre d'une recherche doctorale menée à l'EHESS : dépouillement d'archives, entretiens auprès de militant.e.s paysan.e.s et d'alliés du mouvement, et observations de réunions publiques de La Vía Campesina. 
familiaux et mouvements de paysans sans terres. Ses membres se définissent comme des « producteurs alimentaires (...) prêts, capables et désireux de nourrir les peuples du monde » et déclarent chercher à « développer la solidarité et l'unité dans la diversité parmi les organisations de petits producteurs », et ce dans un but de justice sociale ${ }^{10}$. L'action collective de La Vía Campesina est essentiellement dirigée contre tous les symboles du néolibéralisme, accusé d'être « la cause principale de l'appauvrissement croissant des agriculteurs et des ruraux en règle générale $\gg^{11}$. Ses adversaires désignés sont donc l'OMC, la Banque mondiale, le FMI et, plus largement, tous « les facteurs économiques et politiques détruisant [les] moyens de subsistance, [les] communautés, [les] cultures et [l'] environnement naturel $\gg^{12}$. Pour ses membres, ce qui distingue La Vía Campesina des autres acteurs, c'est qu'elle est avant tout un « mouvement ». Plutôt que de catégoriser les acteurs selon des définitions préconçues, il convient de souligner, comme le rappelle Pierre Bourdieu, que les principes de di-vision sont «toujours subordonnés à des fonctions pratiques et orientés vers la production d'effets sociaux $\gg^{13}$. Il convient ainsi de prêter plutôt attention aux stratégies de distinction par rapport au champ politique, ainsi qu'aux enjeux symboliques que porte telle ou telle labellisation, celle de «mouvement social » pouvant traduire une volonté de se présenter comme un front organisé, uni et contestataire.

Au cours de nos entretiens, les militant.e.s de La Vía Campesina insistent régulièrement sur le caractère inédit de leur « mouvement » qui, selon eux, a permis pour la première fois aux paysans de parler de leur propre voix sur la scène internationale. L'identité de La Vía Campesina réside tout entière dans le refus très net de la part des militants qui ont participé à sa création de voir la cause paysanne plaidée par des «non-paysans ». En effet, bien avant la naissance du mouvement, certaines organisations disposant d'un statut consultatif auprès des Nations unies ou d'autres instances internationales ont eu la possibilité de s'y faire entendre en tant que « représentantes de la société civile » et, ce faisant, d'y parler au nom des paysans, ce qui, aux yeux des représentants paysans, les rendait suspectes de paternalisme. Un bref retour sur la réunion fondatrice de mai 1993 à Mons et sur les deux années de collaboration houleuse (1993-1995) qui s'ensuivirent avec la Fondation Paulo Freire (Paulo Freire Stichting, ci-après PFS), une ONG de développement hollandaise, permet de mieux comprendre l'importance des enjeux pour un certain nombre d'organisations paysannes. Le nom même du mouvement tire son origine d'un programme mis en œuvre dans les années 1980 au Nicaragua par la PFS. D'après les documents de l'époque, il s'agissait de définir, à partir de l'exemple sandiniste,

10. La Vía Campesina, La souveraineté alimentaire pour l'Afrique : un défi à portée de main, 2008, p. 20.

11. La Vía Campesina, Déclaration de Tlaxcala concernant la Vía Campesina, 8 octobre 1996, non paginé.

12. Ibid.

13. Pierre Bourdieu, « L’identité et la représentation. Éléments pour une réflexion critique sur l'idée de région », Actes de la recherche en sciences sociales, 35, 1980, p. 65. Bourdieu utilise les italiques et le tiré pour marquer la notion de visions concurrentes. 
un modèle alternatif, une « voie paysanne $»^{14}$, reposant sur le principe d'une participation accrue des paysans au développement économique rural. Or la réunion de Mons, organisée et financée par la PFS, révéla au grand jour des divergences d'intérêts fondamentales entre cette ONG et les organisations paysannes. Les membres de la PFS projetaient l'inclusion de ces dernières au sein de la Fédération internationale des producteurs agricoles $(\mathrm{FIPA})^{15}$, dominée par les représentants des producteurs les plus intégrés à l'agriculture d'entreprise ou d'exportation, ainsi que l'instauration d'un programme de recherche international mené conjointement par l'ONG hollandaise et par les paysans afin de solliciter des fonds et de promouvoir des projets de développement rural. Les représentants des organisations paysannes, eux, nourrissaient d'autres projets. À l'issue de la réunion, certains décidèrent de créer un mouvement de résistance au néolibéralisme et de construire une « voix paysanne » indépendante de la FIPA et, surtout, de toute autre organisation aspirant à parler au nom et à la place des paysans. Paul Nicholson, membre du syndicat basque Euskal Herriko Nekazarien Elkartasuna et membre fondateur de La Vía Campesina qui a beaucoup œuvré à sa coordination de 1993 à 1996, souligne la violence de cet acte fondateur d'émancipation des organisations paysannes vis-àvis de la tutelle de la PFS : «Avec eux [la PFS], nous avons eu une confrontation très dure, avec une rencontre dans le bureau de l'Institut Paulo Freire où nous nous sommes séparés totalement, et Vía Campesina a pris sa propre responsabilité sur la coordination, sur la direction... sans aucune censure ou... appui. (...) Cette ONG voulait que Vía Campesina soit un espace de réflexion et non un mouvement d'organisations qui prenne des décisions politiques. (...) Nous, nous disions que nous étions responsables de nous-mêmes, que nous prendrions nos décisions politiques et que notre aspiration était de transformer la société $\gg^{16}$.

Plus de vingt ans après, cette rupture demeure prégnante pour un certain nombre de militant.e.s, y compris pour celles et ceux qui n'étaient pas là au moment des faits : une déléguée du Comité de coordination international âgée d'une quarantaine d'années et installée en agriculture à partir du milieu des années 1990, membre de la Confédération paysanne et représentante du mouvement pour l'Europe dans les années 2000, explique ainsi que La Vía Campesina est née avant tout «pour se libérer du joug des ONG » et créer « une organisation paysanne indépendante » qui « parle de manière autonome $\gg^{17}$.

14. Kees Blokland (à l'époque coordinateur des relations internationales de la PFS), « The Peasant Road towards an Alternative Development Model: The Managua Declaration », Development in Practice, 3 (1), février 1993, p. 61-63.

15. Créée en 1946 dans le but de seconder l'Organisation des Nations unies pour l'alimentation et l'agriculture (FAO), elle-même créée le 16 octobre 1945 à Québec, la FIPA, basée à Paris, revendiquait, au moment de sa mise en liquidation judiciaire en novembre 2010, 120 organisations membres issues de 82 pays. Depuis 2011, elle renaît de ses cendres sous le nom d'Organisation mondiale de l'agriculture (OMA), et son siège est désormais situé à Rome. 16. Entretien, Espagne, 2010.

17. Propos recueillis lors d'une journée de formation à la souveraineté alimentaire organisée au siège de la Confédération paysanne, Bagnolet, 2009. 
Cette volonté, en réalité souvent réciproque, de prise de distance et de démarcation entre acteurs sociaux revendiquant soit leur statut de mouvement social, soit leur appartenance à une $\mathrm{ONG}^{18}$, est révélatrice du caractère plus ou moins conflictuel de l'espace dans lequel se tissent les relations entre acteurs transnationaux, « un espace de concurrences et d'alliances, de circulation de ressources et de jeux de légitimation croisés $\gg^{19}$. Les mouvements sociaux peuvent constituer pour les ONG une « caisse de résonance » qui permet à ces dernières de renforcer l'efficacité et la légitimité de leur action. De leur côté, les ONG apportent aux mouvements sociaux une « caution morale » qui leur donne accès à des financements qu'ils ne sont pas souvent en mesure d'obtenir par eux-mêmes. Reste que les alliances conclues entre ces deux catégories d'acteurs sociaux ne sont pas dénuées d'enjeux de pouvoir et d'inégalités, en matière surtout de ressources humaines et financières, d'accès aux rassemblements du mouvement altermondialiste et de visibilité au sein des forums sociaux, par exemple. La déléguée citée plus haut exprime ainsi un sentiment d'《étouffement » dû au fait que, contrairement à de nombreuses autres organisations internationales, le mouvement n'a pu mandater que trop peu de délégué.e.s lors du Forum social mondial de Belém (Brésil, 2009) et que ces derniers (dont elle faisait partie) n'ont pu, par conséquent, contribuer activement aux débats portant sur des thématiques liées à la cause paysanne. Pour toutes ces raisons, le risque d'« ONGisation » du mouvement (selon les propres termes des milititant.e.s) constitue toujours un puissant repoussoir au sein de La Vía Campesina ${ }^{20}$. Sur les « défis relatifs au fonctionnement interne » définis lors de la Ve Conférence internationale du mouvement (Maputo, Mozambique, 2008), on pouvait lire : « Nous avons besoin de renforcer la direction de l'organisation, d'améliorer la formation de notre personnel et des bénévoles, et d'améliorer leur intégration au mouvement en évitant que des "mécanismes du style ONG" n'influent sur notre mode de fonctionnement $\gg^{21}$.

En fait, les relations de La Vía Campesina avec les organisations internationales travaillant sur des thématiques liées à l'agriculture ou au monde rural sont ambivalentes : pleines de méfiance au départ, elles évoluent parfois vers une coopération. Dans les positions politiques officielles du mouvement, il est de plus en plus fréquent d'entendre évoquer la nécessité, au nom d'un certain pragmatisme, d'« alliances stratégiques », à condition toutefois que celles-ci se déroulent dans le cadre de rapports véritablement égalitaires et respectueux de l'autonomie des organisations paysannes. La singularité des acteurs qui prennent

18. Sur la diversité des statuts des ONG, voir William F. Fisher, « Doing Good? The Politics and Antipolitics of NGO Practices », Annual Review of Anthropology, 26, 1997, p. 439-464.

19. Nonna Mayer, Johanna Siméant, «L'espace de l'altermondialisme », Revue française de science politique, 54 (3), 2004, p. 377.

20. Hélène Baillot montre que les mêmes logiques sont à l'œuvre dans le cas de Jubilé 2000. Voir sa contribution dans ce dossier : « La carrière du plaidoyer anti-dette au sein de Jubilé USA. Controverses et (re)définition des "bonnes pratiques" militantes ».

21. Documents politiques de La Vía Campesina, Ve Conférence internationale, 2009, p. 60. 
part à La Vía Campesina - des organisations paysannes pour la plupart, et plus précisément des syndicats - contribue à créer une forme de distanciation, voire de résistance vis-à-vis de l'advocacy tel qu'il est pratiqué de manière croissante, à savoir par des militant.e.s se trouvant souvent « dans la position la plus éloignée de certains de ceux dont ils entendent défendre la cause $\gg^{22}$. Malgré cela, il convient d'examiner de quelle manière la volonté de légitimer et de consolider la dimension internationale de la cause paysanne a conduit les membres de La Vía Campesina à emprunter, avec le concours de certaines ONG et autres « alliés $»^{23}$, des formes de mobilisation plus discrètes et expertes que les démonstrations visibles et fortement publicisées de l'action collective (campagnes, manifestations, rassemblements, occupations... $)^{24}$.

\section{Une stratégie de plaidoyer en faveur des «droits paysans » : opportunités et contraintes}

L'adoption récente, et moins visible que d'autres modes plus traditionnels, de stratégies d'action expertes, souvent regroupées sous le nom de plaidoyer, constitue un tournant dans le répertoire d'action de La Vía Campesina ${ }^{25}$. L'investissement progressif du mouvement dans les sphères onusiennes afin de faire adopter une «Convention internationale sur les droits des paysannes et des paysans » en constitue un exemple significatif.

L'apparition de la thématique des « droits des paysans » au sein de La Vía Campesina découle de discussions politiques portant sur les droits humains qui ont eu lieu lors de la III ${ }^{\mathrm{e}}$ Conférence internationale du mouvement (Bangalore, Inde, 2000). C'est en effet à l'issue de cette conférence qu'a été expressément mise au point une stratégie de plaidoyer en faveur de nouveaux instruments juridiques internationaux spécifiques pour les paysan.ne.s.

L'initiative de cette stratégie de plaidoyer déjà extrêmement cadrée et précise dans ses objectifs trouve en grande partie son origine en Asie du Sud-Est, et plus particulièrement en Indonésie ${ }^{26}$. Le recouvrement des libertés syndicales à partir de 1998 a en effet permis aux organisations paysannes de ce pays de sortir de la

\footnotetext{
22. J. Siméant, « Des mouvements nouveaux et globaux ? Sur les mouvements sociaux "transnationaux" dans quelques ouvrages récents », VIII ${ }^{e}$ Congrès de l'Association française de science politique, table ronde « Où en est la sociologie des mouvements sociaux? », 15-18 septembre 2005, p. 32 .

23. Nous reprenons ce terme employé couramment par les membres de La Vía Campesina pour désigner, par opposition aux « adversaires »du mouvement, les sympathisants éventuels de la cause paysanne avec lesquels ils sont susceptibles de nouer des «alliances ».

24. Voir la contribution de Étienne Ollion dans ce dossier : «Des mobilisations discrètes : sur quelques transformations de l'action collective contemporaine $\gg$.

25. Sur l'importance qu'a représentée l'élaboration de la notion de « souveraineté alimentaire » dans l'internationalisation de la cause paysanne, voir Delphine Thivet, « Des paysans contre la faim », Terrains \& travaux, 20, 2012, p. 69-85.

26. M. Edelman, « Linking the Rights of Peasants to the Right to Food in the United Nations », art. cité, p. 8-9.
} 
clandestinitée ${ }^{27}$ et de se renforcer avec notamment le soutien et la participation d'un certain nombre de militant.e.s des droits humains, du mouvement étudiant et de celui de l'indépendance du Timor oriental. C'est donc dans un contexte national de renouveau des luttes agraires, mais aussi de débats très importants portant sur la réforme agraire qu'est apparue pour la première fois la question des «droits des paysans ». Dans un article consacré aux débats relatifs aux lois de cette réforme dans l'Indonésie de la Reformasi ${ }^{28}$, Anton Lucas et Carol Warren soulignent le rôle important qu'ont joué les ONG et les mécènes étrangers dans l'importation et la diffusion du « discours des droits » dans les luttes agraires en Indonésie au cours des années 1980-1990²9.

En 2001, la Fédération des syndicats paysans indonésiens (FSPI), membre de La Vía Campesina depuis 1996, a organisé une rencontre sur le thème de la réforme agraire (National Conference on Agrarian Reform to Protect and Implement the Rights of Peasants, Cibubur, 17-20 avril 2001). Il en est ressorti une Déclaration des droits fondamentaux des paysans indonésiens, divisée en huit sections et comprenant plus de 67 articles, qui a servi de base au texte rédigé par La Vía Campesina en 2009. Comme le rappelle l'un des fondateurs du Consortium pour la réforme agraire (Konsorsium Pembaruan Agraria/KPA), ONG indonésienne créée en 1994 entre autres pour lutter contre la déforestation et contre l'accaparement des terres agricoles par des entreprises productrices d'huile de palme, cette première Déclaration était elle-même le fruit d'un long travail entrepris depuis 1998 par une coalition rassemblant des professeurs d'université, des organisations paysannes et des ONG revendiquant la mise en œuvre de la réforme agraire en Indonésie ${ }^{30}$. On retrouve au niveau national la même concurrence entre plaideurs de la cause paysanne qu'au niveau internationa ${ }^{31}$. De nombreux débats agitent en effet la sphère militante indonésienne au début des années 1990. Les discussions portent sur les limites de l'advocacy - souvent accompli par des étudiants ou des militants d'origine urbaine, de manière ponctuelle et au gré des financements obtenus de l'étranger - et, par conséquent, sur la nécessité de créer des organisations paysannes autonomes ${ }^{32}$. De fait, la FSPI ${ }^{33}$ est elle-même issue d'une scission qui a eu lieu en 1998 au sein

27. Les militants communistes et les paysans qui avaient bénéficié de la réforme agraire mise en place par Sukarno en 1960 (Basic Agrarian Law) firent l'objet d'une répression sanglante, surtout en 1965-1966 sous la dictature de Suharto.

28. Nom donné au mouvement de « réforme » qui a suivi la chute de Suharto en 1998.

29. Anton Lucas, Carol Warren, « The State, the People, and Their Mediators: The Struggle over Agrarian Law Reform in Post-New Order Indonesia », Indonesia, 76, 2003, p. 99.

30. Dianto Bachriadi, «Between Discourse and Action: Agrarian Reform and Rural Social Movements in Indonesia post-1965 », thèse de doctorat, Flinders University, 2011, p. 271-272.

31. Nancy Lee Peluso, Suraya Afiff, Noer Fauzi Rachman, « Claiming the Grounds for Reform: Agrarian and Environmental Movements in Indonesia », Fournal of Agrarian Change, 8 (2-3), 2008, p. 377-407.

32. Notamment à cause de l'absence de leadership paysan et de la dépendance des paysans vis-à-vis de militants ex-étudiants issus de la classe moyenne urbaine.

33. La FPSI est devenue en 2007 le Syndicat des paysans indonésiens (SPI). Elle a hébergé à Jakarta de 2004 à 2013 le secrétariat international de La Vía Campesina. 
du KPA jugé - notamment par Henry Saragih ${ }^{34}$, président de la FSPI - comme étant dominé par «les $\mathrm{ONG} »^{35}$. Paradoxalement, et alors que la bataille pour une réforme législative au niveau national se révèle peu productive, c'est la FSPI qui se réapproprie la notion de «droits des paysans » et l'importe au sein de La Vía Campesina, impulsant ainsi une stratégie de plaidoyer en faveur de ces derniers au niveau international.

La mobilisation de La Vía Campesina en faveur d'une « Convention internationale sur les droits des paysannes et des paysans » s'étant étalée sur plus de dix ans, nous n'en détaillerons pas chaque étape, mais soulignerons plutôt les techniques spécifiques qu'il a fallu mobiliser pour faire reconnaître par les instances onusiennes la nécessité d'un nouvel instrument de droit international en faveur des paysan. ne.s : d'une part, la production, la mise en forme et la diffusion de témoignages sur la violation des droits humains, d'autre part, un travail de lobbying auprès des représentants des États et des membres des institutions internationales. Si les délégué.e.s de La Vía Campesina ont pu apprendre et s'approprier ces techniques, c'est notamment par leurs interactions avec des ONG engagées dans la défense des droits humains et avec des acteurs travaillant directement au sein des instances onusiennes, notamment dans l'équipe de conseillers et de juristes proches du Rapporteur spécial des Nations unies pour le droit à l'alimentation ${ }^{36}$. Outre la mobilisation des ressources locales et nationales de ses propres membres - principalement en Indonésie et en Suisse (grâce à la forte implication des membres du syndicat UNITERRE qui a un siège à Genève, c'est-à-dire au plus près des instances onusiennes) -, La Vía Campesina a en effet bénéficié du soutien du Centre Europe-Tiers Monde (CETIM), ONG tiers-mondiste située également à Genève, ainsi que du FoodFirst Information and Action Network (FIAN). Empruntant à Amnesty International la technique du «name and shame » qui a fait ses preuves dans le domaine des droits civils et politiques, le FIAN a notamment mis en œuvre une expertise et un soutien juridique dans les cas de violations du droit à l'alimentation dans le monde, lancé des campagnes de protestation par lettres envoyées à l'État violateur, publié des rapports et des documentations... C'est ainsi en étroite collaboration avec le FIAN que La Vía Campesina s'est engagé, en particulier de 2004 à 2006, dans un travail de recensement et de dénonciation systématique des violations « des droits humains des paysans $»^{37}$.

\footnotetext{
34. Ironiquement, Henry Saragih, qui a été par ailleurs le coordinateur général de La Vía Campesina de 2004 à 2013, a commencé à militer comme étudiant à l'Université du Nord Sumatra puis dans une ONG spécialisée dans l'advocacy en faveur des paysans et du développement rural (Yayasan Sintesa, fondée en 1987).

35. D. Bachriadi, «Between Discourse and Action: Agrarian Reform and Rural Social Movements in Indonesia post-1965 », cité, p. 214 ; A. Lucas, C. Warren, « The State, the People, and Their Mediators: The Struggle over Agrarian Law Reform in Post-New Order Indonesia », art. cité, p. 101-102.

36. Jean Ziegler (2000-2008); Olivier de Schutter (2008-2014).

37. La Vía Campesina/FIAN, Violations of Peasants' Human Rights. A Report on Cases and Patterns of Violation 2004 ; Annual Report on Violation of Peasants Rights 2005 ; Annual Report. Violations of Peasants' Human Rights. A Report on Cases and Patterns of Violence 2006.
} 
Outre ces enquêtes militantes et cette mise en forme juridique des injustices subies par les paysans, les délégué.e.s de La Vía Campesina - initiés aux arcanes du système onusien par leurs alliés au sein de l'équipe de conseillers mentionnée plus haut - se sont engagé.e.s dans un travail de lobbying auprès des représentants des États aux Nations unies à Genève pour que ces derniers soutiennent les études préliminaires du Comité consultatif du Conseil des droits de l'homme et, plus largement, le projet d'une Convention sur les droits des paysannes et des paysans. Un tableau classant et identifiant le plus précisément possible les États considérés comme les plus « influents » au sein du Conseil des droits de l'homme, au regard de l'expérience des sessions antérieures, et faisant état de la meilleure manière de sensibiliser leurs représentants à la cause des « droits des paysans » a ainsi été conçu par un membre du Syndicat des paysans indonésiens spécialement chargé de superviser les activités de lobbying de La Vía Campesina à l'ONU. Parallèlement, un appel a été lancé en 2012 à l'ensemble des organisations du mouvement pour qu'elles fassent pression sur leurs gouvernements respectifs. Une stratégie spécifique a été fixée à l'intention de l'Union européenne dont les États membres sont majoritairement opposés à ce projet de convention internationale.

Malgré la « petite victoire $»^{38}$ que représente l'annonce, en septembre 2012 à Genève, de la mise en place d'un groupe de travail intergouvernemental chargé de négocier puis de présenter au Conseil des droits de l'homme un projet de « Déclaration des Nations unies sur les droits des paysans et autres personnes vivant dans les zones rurales », le coût d'entrée pour un mouvement social dans cette forme de plaidoyer faisant étroitement appel à des formats juridiques se trouve interrogé par les militants eux-mêmes.

D'un côté, le recours au droit sur lequel repose cette stratégie constitue indéniablement, dans le processus de mobilisation de La Vía Campesina, une ressource symbolique contre ses adversaires : le discours des droits humains ouvre en effet une arène de dénonciation et une « fenêtre d'opportunité discursive $»^{39}$ visant stratégiquement à délégitimer le « droit de l'OMC » ou plus généralement la lex mercatoria et autres dispositifs juridiques défavorables aux petits paysans. Le capital symbolique attaché aux droits humains fonctionne de fait comme un extraordinaire vecteur de généralisation et de publicisation - voire d'universalisation - de la cause défendue par un mouvement social. Face aux contraintes pesant sur la capacité des acteurs à rendre intelligible et, plus précisément, audible, la cause qu'ils veulent défendre ${ }^{40}$, ce discours se révèle de plus particulièrement efficace pour attirer des financements internationaux et capter l'attention des médias. Toutefois, pour La Vía Campesina, le droit ne se réduit pas à un répertoire de ressources, il contribue

38. La Vía Campesina, «Victory in Hard-Fought Struggle in Defense of the Human Rights of Peasants », communiqué de presse, 5 octobre 2012.

39. Myra Marx Ferree, « Resonance and Radicalism: Feminist Framing in the Abortion Debates of the US and Germany », American fournal of Sociology, 209 (2), 2003, p. 309.

40. Annie Collovald, Brigitte Gaïti, « Des causes qui “parlent”.... » Politix, 4 (16), 1991, p. 7-22. 
indéniablement à accroître la « conscience des droits » chez ses membres ${ }^{41}$ et fournit, comme le souligne Daniel Cefaï, des « opérateurs de cristallisation d'identités individuelles et collectives » contribuant à faire « émerger de nouveaux groupes sociaux (...), [et à les inscrire] dans l'horizon des politiques publiques $\gg^{42}$. D'un autre côté, le choix de cette stratégie suppose la médiation d'un certain nombre d'alliés, des plaideurs de causes professionnels membres d'ONG aux spécialistes du droit international relatif aux droits de l'homme, en passant par des anthropologues ${ }^{43}$ et des fonctionnaires internationaux (notamment les représentants de la Bolivie, de l'Afrique du Sud, de Cuba et de l'Équateur). Malgré des tentatives de subversion du droit, autrement dit de mise en ouvre d'un « double jeu de respect et de torsion des règles du jeu $\gg^{44}$, mêlant à la fois résonance et radicalisme et essayant d'étendre la notion de droits au-delà des normes juridiques existantes ${ }^{45}$, les membres de La Vía Campesina demeurent étroitement dépendants de leurs alliés pour formaliser juridiquement leur cause. Certain.e.s au sein du mouvement expriment en particulier leur défiance à l'égard du droit, conçu généralement comme un instrument de domination sociale : avoir recours au droit, si tant est qu'on puisse y avoir socialement accès, c'est utiliser une langue forgée par d'autres, c'est, de manière hétéronome, se compromettre dans un jeu dont on n'a pas écrit les règles. Une jeune agricultrice française du mouvement exprime sa gêne à l'égard de cette stratégie juridique, et plus largement ses doutes quant à l'utilité du travail de plaidoyer auprès des instances onusiennes ${ }^{46}$ : « Je suis mal à l'aise avec les droits de l'homme. Je trouve qu'il y a une déconnexion entre le langage et la réalité et que les droits de l'homme, ça sert à ça : à exprimer des principes que, de toutes les façons, on ne respecte pas. (...) Pour moi, c'est un langage au-delà du réel. (...) On n'a rien à attendre de la FAO ou de l'ONU.

41. Au sens de Michael W. McCann, Rights at Work: Pay Equity Reform and the Politics of Legal Mobilization, Chicago, University of Chicago Press, 1994.

42. Daniel Cefaï, Pourquoi se mobilise-t-on ? Les théories de l'action collective, Paris, La Découverte/MAUSS, 2007, p. 536-537.

43. L'anthropologue chilien José Antonio Bengoa Cabello, membre du Comité consultatif du Conseil des droits de l'homme, a soutenu le projet de La Vía Campesina (« Declaración Internacional de los Derechos Campesinos », Le Monde Diplomatique (édition cbilienne), mars 2012). M. Edelman, quant à lui, a été étroitement associé au projet de Déclaration sur les droits paysans depuis 2011 et est notamment intervenu en tant qu'expert à la demande du Bureau du Haut-Commissariat aux droits de l'homme lors de la première session du Groupe de travail intergouvernemental pour une « Déclaration des Nations unies sur les droits humains des paysans et des autres personnes travaillant dans les zones rurales » (Genève, juillet 2013). M. Edelman, « What Is a Peasant? What Are Peasantries? A Briefing Paper on Issues of Definition », 2013.

44. D. Cefaï, Pourquoi se mobilise-t-on? Les théories de l'action collective, op. cit., p. 490.

45. La Déclaration de La Vía Campesina mentionne en effet à la fois des droits déjà reconnus par le système du droit international (l'égalité homme-femme, la non-discrimination, le droit à l'eau et à l'alimentation, à un logement, à la liberté d'expression...) et des revendications plus politiques telles que le « droit à la réforme agraire », le «droit à un juste prix pour sa production », le «droit aux semences », le «droit à participer aux décisions $\gg$.

46. Sentiment d'ailleurs relativement répandu parmi les militant.e.s d'Amérique du Sud où les violations quotidiennes de l'État de droit, par-delà la façade juridique existante, sont importantes. Voir notamment Paulo Sérgio Pinheiro, « Démocratie et État de non-droit au Brésil : analyse et témoignage », Cultures \& Conflits, 59, automne 2005, p. 87-115. 
La force des mouvements sociaux, c'est avant tout leur capacité de résistance, leur capacité à bloquer des processus qui produisent toujours plus d'injustices. Ce ne sont pas les institutions internationales qui vont régler les problèmes des paysans. (...) Ce qui est très fort, c'est l'unité des problèmes rencontrés par les paysans sur la planète, aussi bien au Nord qu'au Sud. C'est le rapport à la nature, le fait de se voir expulsé d'une terre, (...) tout ça, ça nous réunit. Et se réunir, construire une unité entre les paysans, c'est plus important que tout le travail institutionnel $\gg^{47}$. Ce scepticisme s'exprime également en termes de calcul des coûts : à quoi bon investir autant - en ressources humaines et matérielles, mais aussi en temps - si les bénéfices escomptés demeurent incertains? Avant d'être adoptée par l'Assemblée générale de l'ONU, la Déclaration des Nations unies sur les droits des peuples autochtones $(2007)^{48}$, qui a servi de modèle à La Vía Campesina pour sa propre Déclaration, a fait l'objet de plus de vingt-cinq ans de travail de mobilisations. Or les conditions de son application sont toujours en débat aujourd'hui. Comme son nom l'indique, ce texte est en effet seulement « déclaratif » et, dans la mesure où il n'est pas accompagné d'une convention qui requiert une ratification des États, il ne comprend aucun mécanisme contraignant (soft law).

\section{Une modération de la cause?}

Les alliances stratégiques nouées par le mouvement et la mise en forme juridique de la cause entraînent un travail de recadrages à répétition qui, certes, peut être considéré comme dynamique et interactionnel, mais qui demeure souvent asymétrique, les ONG disposant de davantage de ressources humaines et financières et étant, de ce fait, moins disposées à modifier leur propre cadrage. En fait, ce processus d'adaptations successives a surtout vocation à rendre la cause acceptable par des alliés dont les intérêts peuvent parfois diverger de ceux du mouvement, notamment lorsque celui-ci se tourne vers des stratégies d'action directe ou extrajuridique). Citons à titre d'exemple les réticences initiales du Comité consultatif du Conseil des droits de l'homme à employer dans ses premiers rapports le terme de «droits des paysans » et la requalification de la proposition initiale de La Vía Campesina en projet seulement déclaratif. En outre, parce qu'elle exige la maîtrise de savoirs et de compétences sociales et techniques qui ne sont pas immédiatement accessibles à tout un chacun, cette forme plus experte qu'est le plaidoyer fait redouter à certain.e.s militant.e.s une accentuation de la division du travail militant international. L'un d'entre eux reconnaît en effet que l'aspiration à acquérir dans les instances onusiennes un «droit de parole » qui ne soit pas médiatisé par des «officiels » ou des ONG, droit revendiqué de longue date,

47. Entretien, France, 2010.

48. Irène Bellier, « Les peuples autochtones aux Nations unies : un nouvel acteur dans la fabrique des normes internationales », Critique internationale, 54, 2012, p. 61-80. 
pose des défis qui engagent la crédibilité du mouvement tant ils peuvent paraître contradictoires : le défi de maintenir une « voix authentique » et un «droit au dissensus » au sein de ces espaces, et celui d'acquérir parallèlement les compétences nécessaires - notamment la grammaire du « parler en public » propre à un univers à dominante diplomatique - pour exercer dans ces mêmes espaces une véritable influence ${ }^{49}$. Dès lors, il conviendrait d'explorer plus en détail la manière dont les activités de plaidoyer contribuent à consolider dans une position d'élite militante un petit groupe de représentants qui, souvent responsables de leur organisation paysanne au niveau national et géographiquement proches des centres de décisions quasiment tous situés au « Nord », se spécialisent progressivement dans des fonctions de lobbying et de « relations publiques » au sein du mouvement. La majorité d'entre eux sont dotés de compétences en communication, capables de s'adapter au formalisme des audiences institutionnelles, de s'exprimer en anglais et de parler avec aisance aux journalistes, diplomates et responsables d'ONG. Leur médiation entraîne des luttes symboliques et des enjeux de pouvoir - par exemple entre une vision experte et la construction «spontanée » du sens de la lutte par les militants -, mais aussi une perte d'autonomie de ces derniers dans la définition de leur cause et la conservation de l'enjeu de leur lutte.

De fait, le plaidoyer exige de plus en plus un apprentissage et des compétences spécifiques que quelques militant.e.s seulement au sein du mouvement sont à même, en l'état actuel des choses, de mettre en œuvre : une parole à la fois experte et policée et la construction de liens avec les autorités des instances internationales. Et cette exigence contraint bien souvent les membres de La Vía Campesina à solliciter des ressources externes auprès de ses alliés, au risque parfois d'une cooptation $^{50}$ et d'une perte de l'autonomie originellement revendiquée en matière de cadrage et de défense de la cause paysanne. Le mouvement ne peut alors échapper à des débats de fond sur la possible dépolitisation ${ }^{51}$ ou « déradicalisation » de la cause. À force d'insister sur le statut de « groupe vulnérable » ou de victimes des paysans ainsi que sur les violations des droits humains et sur la discrimination dont ils font l'objet, les catégories juridiques prennent, semble-t-il, le pas sur le langage plus politique de désignation des « coupables » et de lutte notamment contre les entreprises multinationales semencières et agro-alimentaires, et autres fonds d'investissement, qui échappent largement à l'emprise du droit international des droits humains. Le travail d'advocacy conduit en outre à un éloignement

\footnotetext{
49. Entretien, Italie, 2013.

50. Jeff Corntassel, « Towards a New Partnership? Indigenous Political Mobilization and Co-optation during the First UN Indigenous Decade (1995-2004)», Human Rights Quarterly, 29 (1), 2007, p. 137-166.

51. James Ferguson, The Anti-politics Machine: « Development », Depoliticization and Bureaucratic Power in Lesotho, Minneapolis, University of Minneapolis Press, 1994.
} 
vis-à-vis de la construction d'alternatives concrètes visant au changement social que revendique par ailleurs le mouvement ${ }^{52}$.

La Vía Campesina n'élude pas ces critiques internes et se garde bien d'y apporter une réponse univoque. L'une de ses publications récentes met particulièrement en relief la difficulté de construire une mobilisation au niveau international et, comme tout mouvement social, de conserver un équilibre entre sa position d'extériorité critique vis-à-vis des lieux de pouvoir et son inscription dans une logique de négociation et de compromis nécessaire à tout travail institutionnel : « La question de l'équilibre à trouver entre mobilisation et travail institutionnel se pose sans cesse. Il n'y a pas de réponse unique car cet équilibre évolue au fil du temps, des contextes politiques, sociaux, économiques, des rapports de force, etc. (...) Comme pour le travail que nous faisons sur la Déclaration des droits des paysans et des paysannes, il y a un juste dosage à trouver et nous sommes loin d'être dans du simple travail de lobbying. Pour agir à l'intérieur [des lieux de pouvoir], il est souvent indispensable de disposer de documents d'une grande qualité technique, afin qu'une faiblesse sur un détail ne vienne pas déstabiliser notre crédibilité politique $\gg^{53}$.

Une piste évoquée au sein du mouvement est de former davantage de militants à la spécificité du travail institutionnel dans des arènes internationales pour donner à un plus grand nombre d'entre eux la possibilité de participer à cette tâche extrêmement ambitieuse mais exigeante que représentent la construction et la consolidation d'un mouvement social transnational.

L'étude de la façon dont les militant.e.s de La Vía Campesina se sont engagé.e.s dans la promotion de « droits paysans » auprès du Conseil des droits de l'homme des Nations unies permet d'observer les modalités de diffusion et d'appropriation par des acteurs initialement réticents à leur égard de formes de mobilisation parfaitement adaptées aux exigences des arènes internationales. Au-delà de la méfiance toujours présente des membres de La Vía Campesina envers des porte-parole trop « paternalistes » à leurs yeux, ce cas de mobilisation original met également en lumière les alliances nécessaires qu'un mouvement social est contraint de nouer pour mettre en œuvre un répertoire initialement récusé tout en veillant à maintenir pour lui-même « des marges de manœuvre et espaces de jeux $\gg^{54}$. Malgré la consécration symbolique forte qu'elle représente pour le mouvement en matière de reconnaissance du travail accompli depuis 2002, la récente décision par le Conseil des droits de l'homme de former un groupe de travail intergouvernemental destiné

52. D. Thivet, « La Vía Campesina et l'appropriation de l'agroécologie », dans Aurélie Cardona, Fanny Chrétien, Benoît Leroux, Fabrice Ripoll, Delphine Thivet (dir.), Dynamiques des agricultures biologiques. Effets de contexte et appropriations, Versailles/Dijon, Quae/Educagri, 2014, p. 75-92.

53. « Le CSA [Comité sur la sécurité alimentaire] : un nouvel espace pour les politiques alimentaires du monde », Les Cabiers de La Vía Campesina, 4, 2012, p. 15.

54. «Militantismes institutionnels », Politix, 70, 2005, p. 4. 
à préparer au sein de l'ONU une Déclaration internationale sur les droits des paysans semble faire entrer La Vía Campesina dans une nouvelle ère de son travail de mobilisation transnationale. Depuis quelques années en effet, le mouvement « s'est engagé sur la voie de la collaboration avec les instances onusiennes $»^{55}$. C'est dans ce contexte d'institutionnalisation que l'advocacy se révèle être une technique particulièrement efficace pour porter de manière discrète à l'intérieur des lieux de pouvoir une cause paysanne maintenue pendant des années au seuil de ces mêmes lieux devant des portes résolument fermées ${ }^{56}$. Cette évolution permet de repenser la dichotomie traditionnelle propre à la sociologie des mouvements sociaux entre insiders et outsiders et invite à analyser de manière plus précise et circonstanciée de quelle manière et à quelles conditions des mouvements tels que La Vía Campesina sont susceptibles d'impulser une «subversion feutrée » au sein de sites institutionnels tels que la FAO et le Conseil des droits de l'homme, comme l'ont fait dans d'autres instances internationales les mouvements de femmes décrits par Laure Bereni et Anne Revillard ${ }^{57}$.

Delphine Thivet est docteure en philosophie de I'Université Paris 1 Panthéon-Sorbonne et doctorante en sociologie au Centre Maurice Halbwachs-équipe PRO et à I'Institut de recherche interdisciplinaire sur les enjeux sociaux (IRIS) (EHESS). Ses recherches actuelles portent sur le processus de transnationalisation des mouvements sociaux. Elle a notamment publié "Des paysans contre la faim. La "souveraineté alimentaire", naissance d'une cause paysanne transnationale », Terrains \& Travaux (20, 2012, p. 69-85) et " Peasants' Transnational Mobilization for Food Sovereignty in La Vía Campesina » dans Carole Counihan, Valeria Siniscalchi (dir.), Food Activism: Agency, Democracy and Economy (Londres, Bloomsbury, 2014, p. 193-209).

delphine.thivet@ehess.fr

55. Coordination européenne-Vía Campesina, Un pas de plus pour une Convention internationale sur les droits des paysannes et paysans, 16 février 2012, lettre de plaidoyer envoyée aux gouvernements nationaux et à leurs représentants à Genève.

56. Lors du Sommet mondial sur l'alimentation organisé par la FAO (Rome, 1996), les représentants de La Vía Campesina s'étaient en effet vu refuser leur demande d'accréditation au sein des lieux de discussion officiels.

57. Laure Bereni, Anne Revillard, « Un mouvement social paradigmatique ? Ce que le mouvement des femmes fait à la sociologie des mouvements sociaux », Sociétés contemporaines, 85, 2012, p. 31. 